Canadian University Music Review

Canadian University Music Review

Revue de musique des universités canadiennes

\title{
Pour un printemps nouveau du Sacre
}

\section{Louis Cyr}

Numéro 2, 1981

URI : https://id.erudit.org/iderudit/1013743ar

DOI : https://doi.org/10.7202/1013743ar

Aller au sommaire du numéro

\section{Éditeur(s)}

Canadian University Music Society / Société de musique des universités

canadiennes

\section{ISSN}

0710-0353 (imprimé)

2291-2436 (numérique)

Découvrir la revue

Citer cet article

Cyr, L. (1981). Pour un printemps nouveau du Sacre. Canadian University Music Review / Revue de musique des universités canadiennes, (2), 39-55.

https://doi.org/10.7202/1013743ar

All Rights Reserved (C Canadian University Music Society / Société de musique des universités canadiennes, 1981
Ce document est protégé par la loi sur le droit d'auteur. L’utilisation des services d'Érudit (y compris la reproduction) est assujettie à sa politique d'utilisation que vous pouvez consulter en ligne.

https://apropos.erudit.org/fr/usagers/politique-dutilisation/ 


\title{
POUR UN PRINTEMPS NOUVEAU DU SACRE
}

\author{
Louis Cyr
}

Avec la parution, sur étiquette Philips (9500 781) au printemps de 1981, du Sacre du printemps inteprété par l'orchestre symphonique de Boston sous la direction de son chef attitré Seiji Ozawa, nous parvenait le soixante-quatrième enregistrement fait sur disque de cette partition d'orchestre depuis que Pierre Monteux, au pupitre lors de sa création scénique le 29 mai 1913, en fit en 1928 la première gravure à Paris sur quatre disques 78-tours de La Voix de Son Maître. ${ }^{1}$

Sans doute que le centenaire de la naissance de Stravinsky en 1982 sera l'occasion d'ajouter à cette discographie plus qu'abondante quelques nouvelles gravures du Sacre, au grand désespoir d'ailleurs des critiques. Car, se diront-ils à nouveau, à part les raffinements ultimes de la très haute fidélité numérique, que peut-on vraiment attendre d'inédit d'une ouvre qui a depuis près de soixante-dix ans sans doute livré tous ses secrets rythmiques et sonores? Et pourtant ...

En 1967 l'éditeur Boosey and Hawkes, acheteur en 1947 de l'ancienne Édition Russe de Musique où fut publiée pour la première fois à la fin de 1921 (ou au tout début de 1922) la partition d'orchestre du Sacre, décida de remplacer les vieilles plaques usées et surcorrigées de 1914/15 - date du seul tirage du jeu d'épreuves - par une gravure entièrement nouvelle de l'œuvre, celle qui porte maintenant le numéro d'édition B. \& H. 19441 (156 pages). Précédant de quatre ans seulement la mort ducompositeur (en avril 1971), cette gravure représentait sans doute - à supposer qu'il l'ait personnellement supervisée - ses dernières volontés sur le Sacre. Et pourtant ... Dès 1972 en effet, Claudio Spies était amené à conclure, à propos de cette gravure, ce qui suit :

Revue de musique des universités canadiennes, $\mathrm{N}^{\circ} 2,1981$ 
Telle quelle, cependant, la nouvelle gravure faite par Boosey and Hawkes en 1967..., en plus de contenir certaines modifications dont l'authenticité est plus que douteuse... laisse sans réponse la question fondamentale de savoir si elle représente entièrement les intentions de Stravinsky, en plus de suggérer une autre question, à savoir : pourquoi on s'est donné la peine de graver ce qui ne saurait être qu'une autre partition bouche-trou de l'œuvre (Spies 1972 : 258). ${ }^{2}$

Jugement sévère mais malheureusement justifié, si l'on examine de près la partition de 1967 en la remplaçant dans le contexte de toutes les (ré-) impressions de l'ancienne gravure. Jugement corroboré de plus, au moins dans les studios d'enregistrement, par le fait que sur vingt-sept gravures sur disque réalisées jusqu'ici depuis 1967, une seule (celle de Simon Rattle avec le National Youth Orchestra sur étiquette anglaise Enigma) reproduit fidèlement la partition de 1967! Jugement confirmé enfin par le fait que cette partition ne tient toujours pas compte de plusieurs détails d'interprétation que l'on retrouve avec une constance certaine dans les trois enregistrements du compositeur lui-même.

Soit, dira-t-on, mais une fois toutes ces divergences et incertitudes résolues, le seul problème à régler est celui d'une édition critique du Sacre permettant de rassembler en une seule interprétation la lecture parfaitement fidèle et irréprochable d'une partition dont, de façon éparpillée ici et là jusqu'ici, on connaîtrait amplement toutes les facettes sonores.

On ne peut nier de fait avoir eu droit jusqu'à aujourd'hui, tant sur disque qu'en salle de concert, à bien des lectures rythmiques différentes et à une variété étonnante de tempi dans toutes les parties du Sacre, une ouvre qui, sur ces plans, pourtant tolère si peu d'écarts. Et cependant, tout n'a pas encore été mis à jour quant au détail de l'articulation rythmique et dynamique et de l'orchestration du Sacre. Car si l'établissement d'une édition critique constitue un défi quasi insurmontable, les sources manuscrites et imprimées accessibles maintenant nous révèlent de nombreux détails dont la modification ou la disparition ultérieure ne peuvent pas toujours être ramenées à une volonté claire et irrévocable du compositeur.

Sans prétendre privilégier la partition dans son revêtement orchestral du 29 mai 1913, il nous est possible de relever plusieurs aspects de détail dont l'audition concrète - et non 
seulement l'authenticité autographe ou imprimée - est susceptible de modifier sensiblement notre perception de l'œuvre, surtout dans le sens d'une articulation plus nerveuse et sèche qui rapproche le Sacre encore davantage de ses origines pianistiques et percussives. Cela apparaît plus nettement dans la Danse sacrale, la dernière scène de l'œuvre qui, avec Glorification de l'élue, constitue le plat de résistance rythmique de toute l'œuvre.

Il y a donc encore place pour des sonorités inouïes dans le Sacre. Et en plus d'entendre bientôt sur disque $\mathrm{x}$ éclairages nouveaux de la partition actuelle avec toutes ses ambiguïtés, nous souhaiterions, pour fins de comparaison, voir quelque chef et orchestre courageux se donner la peine de faire résonner quelques-unes au moins de ces sonorités d'origine. Non pas pour faire plus " authentique " ni pour le plaisir d'une simple restauration puriste, mais plutôt pour nous enrichir du potentiel sonore toujours enfoui dans la partition et dont le secret n'est pas seulement imputable aux imperfections de nos instrumentistes, de nos appareils reproducteurs ou de nos salles de concert. Qui sait, peut-être d'ici 1988 - le 75e anniversaire de la création du Sacre - un éditeur de disques sera preneur, et nous pourrons alors comparer à loisir ...

\section{Manuscrits et partitions en présence}

Sans vouloir entrer ici dans tout le détail, ${ }^{3}$ signalons d'abord les documents manuscrits suivants :

- la collection d'esquisses d'André Meyer publiée en 1969 par Boosey \& Hawkes;

- le manuscrit autographe du Sacre, actuellement la propriété du docteur Paul Sacher de Pratteln (près de Bâle, en Suisse), et dont une copie sur microfilm peut être consultée à la bibliothèque municipale de New York (Lincoln Center);

- une copie manuscrite de cet autographe effectuée à Leipzig fin-avril et début-mai 1913 par un copiste (O.Th.) de l'imprimeur C.G. Roeder; cette copie, conservée chez Boosey and Hawkes à Paris, servit directement à la gravure du jeu d'épreuves en 1914/15;

- les parties manuscrites (de plusieurs copistes) des vents et percussions transcrites d'après la première partition publiée en 1921/22; ces parties sont en dépôt chez l'éditeur Boosey à Londres ;

- des pages manuscrites de Stravinsky et d'Ernest 
Ansermet concernant la Danse sacrale et l'Évocation des ancêtres telles que révisées et nouvellement gravées en 1929; ces pages manuscrites sont conservées pour la plupart à la bibliothèque du Conservatoire de Musique de Genève. Les deux premières pages de la Danse sacrale ainsi ré-écrites par Stravinsky sont reproduites dans Stravinsky-Craft 1978 : 80-81;

- le manuscrit autographe de la Danse sacrale telle que révisée en 1943, dont le compositeur fit cadeau à Nadia Boulanger et qui est conservé maintenant au Département de la Musique de la Bibliothèque Nationale à Paris.

Le matériel imprimé se divise en trois catégories :

a) La réduction pour piano à quatre mains par l'auteur, la première partition du Sacre à être publiée dès la mi-mai 1913 chez l'Édition Russe de Musique, laquelle la réimprima telle quelle dès 1914 et ensuite en 1922 ; cette partition ne subira de corrections pour la première fois qu'en 1968, lors de la troisième réimpression faite par Boosey and Hawkes pour la rendre conforme (mais en partie seulement) à la partition d'orchestre de 1967.

b) La partition d'orchestre, laquelle fut publiée pour la première fois par l'Édition Russe fin 1921 ou début 1922 en format folio tout comme en format de poche. Portant déjà ellemême la marque de très nombreuses corrections par rapport au jeu d'épreuves de 1914/15. (dont il demeure malheureusement impossible aujourd'hui de trouver un seul exemplaire), cette partition se verra très tôt accompagnée en encart d'un avertissement détaillé de deux pages destiné au chef d'orchestre et d'une liste d'errata de trois pages, rédigés tous les deux par Ernest Ansermet en étroite collaboration avec le compositeur. Suite à de nouvelles modifications rythmiques d'envergure, dont on ne sait toutefois s'il faut les attribuer plutôt à Ansermet qu'à Stravinsky, on dût dès 1929 graver à nouveau l'Évocation des ancêtres ainsi que le début (et sa reprise) et la fin de la Danse sacrale. Ces pages refaites furent incorporées à la réimpression faite cette année-là de la partition de poche. Après la publication séparée chez Associated Music Publishers à New York en 1945 d'une toute autre version de la Danse sacrale entièrement révisée par l'auteur, Boosey and Hawkes réimprimèrent en 1948 les deux partitions, grande et petite, de l'Édition Russe du Sacre en y incorporant la correction des erreurs signalées à part en 1922. Quinze tirages de la partition de poche ainsi corrigée verront le jour chez Boosey avant 1967, dont celui 
de la fin de 1951 dans lequel on dut effectuer plus de trois cents autres corrections de détail. Seule une liste dactylographiée de ces dernières fut glissée après 1950 dans la grande partition, laquelle ne fut corrigée en conséquence que lors de la réimpression en 1965. A peine deux ans plus tard, face à l'usure des plaques anciennes et confronté à toute une batterie de nouvelles corrections, l'éditeur Boosey procèdera - comme nous l'indiquions au début de cet article - à une gravure toute nouvelle de la partition, non sans y intégrer finalement de nouvelles modifications dont l'authenticité peut être sérieusement mise en doute.

c) Le matériel d'orchestre, dont la publication poursuivit une trajectoire différente et encore plus bizarre que les deux premières. Ce n'est en effet qu'en 1926-29 que furent gravées en notation d'imprimerie par l'Édition Russe les parties d'orchestre, et c'est de ces mêmes plaques, diversément corrigés, qu'on a tiré les trois réimpressions faites depuis lors chez Boosey (en 1950 à Paris, en 1965-66 et en 1971 à Londres). Car seules les cordes semblent avoir été gravées à la main en 1913, le reste du matériel ayant été seulement copié à la main. Non seulement la guerre de 1914 aura ensuite empêché d'en préparer une gravure définitive, mais même en 1922, l'Édition Russe n'aura fait faire de tout le matériel d'orchestre qu'une nouvelle gravure manuelle. De plus, tout au long de ce périple fort mouvementé, cordes et vents (et percussions) semblent avoir été traités séparément et de façons différentes. Pis encore, ni l'Édition Russe ni Boosey ne réussirent à rapatrier à chaque étape tout le matériel d'orchestre, soit pour le corriger uniformément, soit pour lui substituer tout simplement un matériel plus neuf. Bien malin alors celui qui parviendrait à décortiquer l'imbroglio incroyable de partitions et de matériels divergents qui, remontant jusqu'aux premières gravures de 1926-29, sont encore en circulation aujourd'hui. À moins qu'il ne réclame explicitement partition et matériel de 1967, le chef d'orchestre non prévenu pourra - comme cela s'est vu encore en 1969 - se trouver en face de la grande partition "révisée » de 1965 avec les cordes de 1950 et les vents-percussions de 1929. Et que dire des quelques chefs d'orchestre (Stravinsky lui-même, Monteux, Ansermet, Markevitch, Koussevitzky, Boulez ...) qui, de tout temps, ont eu à leur disposition un matériel réservé en exclusivité à eux seuls ... ? Et nous faisons abstractions ici des 
partitions et matériels parus chez d'autres éditeurs aux U.S.A. et en U.R.S.S. ${ }^{4}$

Les divergences que l'on peut constater entre versions enregistrées sur disque se rattachent donc aussi à cette multiplicité incontrôlée de matériels imprimés en circulation. Il est alors facile d'imaginer la tâche extrêmement ardue qu'aurait à affronter celui qui se verrait confier une édition critique du Sacre, à laquelle Stravinsky lui-même ne s'est au fond jamais vraiment intéressé. Car nous faisons face à une situation qui reflète un peu l'insouciance du compositeur et du chef d'orchestre Stravinsky. Celui-ci, en effet, n'était par nature pas du tout enclin à se complaire indéfiniment dans ses partitions antérieures, une fois celles-ci achevées et créées; il n'hésitait pas à effectuer des modifications sur place lors de répétitions qu'il dirigeait, quitte à les annuler le lendemain ; il a semblé toujours profondément ennuyé et contrarié par des erreures relevées par les instrumentistes. Mais il a fait surtout et pendant longtemps une confiance presque aveugle à Pierre Monteux et avant tout à Ernest Ansermet, eux chefs d'orchestre expérimentés, qui n'ont pas manqué d'attirer son attention sur telle ou telle faiblesse d'instrumentation momentanée ou chronique de tel ou tel passage du Sacre et de ses autres cuvres. Jusqu'au jour où, pour toutes sortes de raisons pratiques et idéologiques, Stravinsky entra en concurrence avec eux et, prenant lui-même la baguette, retrouva, parfois même inconsciemment, le chemin de ses intuitions premières ...

Quoi qu'il en soit, la confrontation de toutes ces sources nous place devant un chassé-croisé continu de modifications, de corrections, de retouches, dont nous voudrions signaler brièvement les plus importantes, d'abord dans toute l'œuvre à l'exclusion de la Dance sacrale que nous examinerons à la fin séparément.

\section{Les principales divergences constatées dans l'ensemble du Sacre.}

Nous les relevons ici dans l'ordre où elles apparaissent dans la partition.

- Dans l'Introduction à la première partie : la reprise un demi-ton plus bas au no. 12 de la célèbre mélodie initiale du premier basson solo doit se terminer par le point d'orgue affectant le la bémol final qui s'éteint imperceptiblement. 


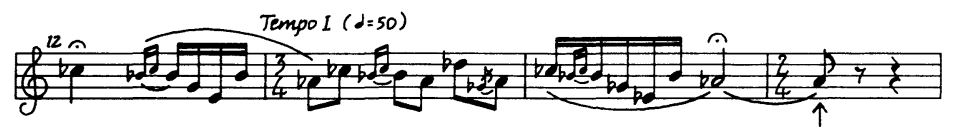

Exemple 1

Le «fa » qu'on y accroche d'autant plus désespérément qu'il est bref (une croche) et très difficile à faire coïncider exactement avec le premier " ré " en pizzicato des premiers violons, est un non-sens modal. Il est vrai que Stravinsky a fait dans son manuscrit autographe une erreur de transposition en clé de do 4 e ligne qu'il n'a par la suite que corrigée partiellement, erreur reproduite ensuite par son copiste à Leipzig et par la graveur de la partition. La réduction pour piano était pourtant sans équivoque là-dessus, du moins jusqu'en 1968, alors qu'on s'avisa de l'aligner sur la partition fautive! Les enregistrements de Stravinsky lui-même, de Monteux, d'Ansermet et de bien d'autres encores sont là pour témoigner de l'authenticité du la bémol tenu.

- Dans les Augures printaniers:

a) dans le manuscrit autographe, dans la copie de Leipzig tout comme dans les parties de cordes de 1913, après le " $p$ sub " qui intervient au no. 19 de la partition il n'y a, à part les accents, aucune variation dynamique dans la répétition (aux cordes en croches) de l'accord célèbre de VI avec $V_{5}^{6}$ superposée en la bémol mineur (harmonique). Donc pas de paliers successifs alternant subitement de " $\mathrm{p}$ » à « $\mathrm{f}$ » ou vice-versa. Les accords accentués se détachent nettement sur le fond d'un simple " martellato" continu en " $p$ ». C'est ainsi d'ailleurs qu'au moins Stravinsky, sinon Monteux, ont toujours interprété ce passage. Pourtant, ces paliers dynamiques successifs alternant entre « $p$ » et « $f$ » se trouvaient dans la réduction pour piano imprimée en 1913 ... Qui aurait rendu conforme à celle-ci en 1922 la partition et le matériel d'orchestre?

b) Il n'est pas du tout certain que le " col legno (battuto) » affecté aux seconds violons après le no. 24 de la partition provienne du compositeur. Cette directive n'apparaît en effet dans le matériel d'orchestre qu'après 1929 et dans la partition d'orchestre qu'en 1951, sans aucune mention préalable ni dans la liste d'errata de $1922 \mathrm{ni}$ dans la correspondance connue Ansermet-Stravinsky. Et on ne saura finalement qu'en 1967 que ce « col legno " doit prendre fin juste avant le no. 30 au lieu 
d'avant le no. 28 , là où s'arrête celui des altos et des violoncelles. Le "battuto" continu de notes différentes de ces seconds violons est d'ailleurs pratiquement inefficace quant à la hauteur des notes, surtout lors des sauts de quinte et de sixte à partir du no. 28. Quelques chefs d'orchestre semblent d'ailleurs ignorer totalement cette directive (Monteux, Bernstein, Ozawa...) et jouent tout simplement " arco ». Ce " col legno " impraticable disparaît de toute façon dans la masse orchestrale assez puissante des nos. 28 et 29.

Il serait par ailleurs fort intéressant d'entendre l'orchestration première de ces mêmes nos. 28-29 de la partition, conservée intacte dans toutes les sources de 1913 mais rayée à grands traits et suivie de la nouvelle version manuscrite de Stravinsky. La mélodie d'accords des quatre trompettes soulignée par trois violoncelles jouant à l'unisson la voix supérieure était confiée à l'origine à quatre cors doublés exactement par quatre altos. Suite aux premières répétitions du Sacre, Pierre Monteux prévint Stravinsky le 30 mars 1913 qu'à moins de faire jouer " pp » le reste de l'orchestre, cette mélodie d'accords resterait inaudible (voir Stravinsky-Craft 1978 : 96). Le compositeur prit acte et modifia radicalement l'orchestration de tout le passage, en profitant même pour y introduire timbales, cymbales antiques et un triangle frappé par une baguette de bois - tous des instruments n'y figurant pas à l'origine! (Les deux derniers n'apparaissent même pas ailleurs dans le Sacre.)

Enfin, des pizzicati de contrebasse doublant, en les accentuant, les violoncelles "arco " aux nos. 32 et 33 ont mystérieusement disparu de la partition et du matériel d'orchestre en 1921 - pourquoi seulement ici, alors que techniquement rien n'y contraint, et non partout ailleurs dans le Sacre? Il s'agit en effet d'un moyen d'articulation mis en œuvre très fréquemment par Stravinsky pour donner plus de mordant aux cordes dont le lyrisme et le vibrato plutôt « romantiques » ne l'attiraient pas du tout à l'époque du Sacre.

- Dans Jeu du rapt : il serait peut-être temps d'ajouter définitivement aux quatre trompettes en début de la 7 e mesure du no. 40 le premier renversement de $\mathrm{V}^{7}$ sur si bécarre à la suite - chromatique descendante - des quatre accords semblables précédents. La correction est faite dans le manuscrit autographe de 1913 déjà, et certains chefs d'orchestre (dont Stravinsky, Monteux, Ansermet, etc.) complètent ainsi la descente, même si, 
dans le matériel d'orchestre, seules la 1re trompette descend au si et la 3e (!) remonte au la.

- Dans Jeux des cités rivales : l'addition d'une deuxième portée de timbales - notes fa-dièse(sol)/do - au début de cette scène figure bien dans la liste d'errata d'Ansermet de 1922.

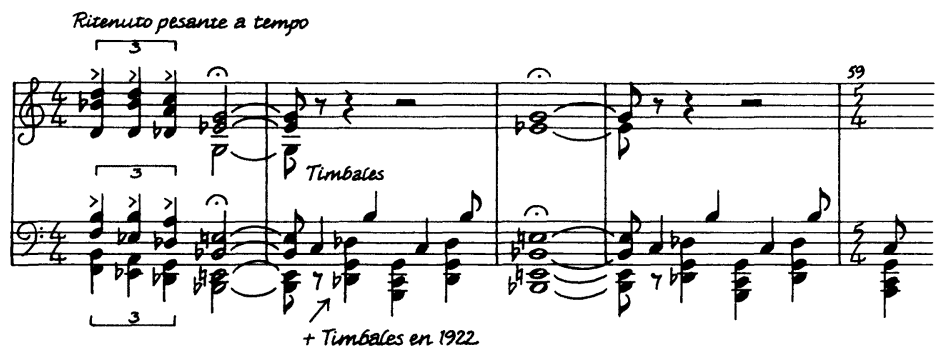

Exemple 2

Robert Craft a même reproduit la page de la partition de poche de 1922 sur laquelle Stravinsky a effectué lui-même en traits rouges une partie de cette correction (p. 48 de l'ancienne gravure) (Stravinsky-Craft $1978: 144$, planche 4e). Outre le fait cependant que cette correction demeura incomplète dans le matériel d'orchestre jusqu'en 1950 et qu'on ne l'introduisit dans la partition d'orchestre qu'en 1948, il semble étonnant de voir Stravinsky brouiller ainsi le lien " thématique » entre cette septième majeure oscillante des timbales (do-si) et sa jumelle du début de Jeu du rapt. Ansermet, responsable de la reprise du Sacre à Londres dès 1920, aura peut-être éprouvé de la difficulté à obtenir des accords de cuivres et de cordes assez percutants pour alterner sur un pied d'égalité avec les coups de timbales et aura suggéré au compositeur de renforcer les premiers moyennant deux timbales additionnelles. C'est un besoin que n'aura pas ressenti Monteux pourtant sept ans auparavant ...

Là n'est toutefois pas la modification la plus étrange à intervenir ici. Les sept notes de l'accord tenu en blanches (ou rondes) confiées, sans doublure aucune (comme celles des accords en triolet) à sept cors, contenaient dès l'origine l'octave stridente de mi bécarre, bien typique de ces frottements de seconde mineure qu'affectionnait Stravinsky. Or dès 1922 (liste d'errata) cette octave est amputée du mi supérieur et réduite au mi inférieur doublé par deux bassons à l'unisson. Le si bémol de la basse est confiée à deux tubas et les quatre notes restantes 
sont doublées chacune par deux cors. Les bassons auront beau jouer "ff ", leur mi bécarre parvient rarement à troubler perceptiblement l'accord rayonnant de sixte et quarte sur si bémol des cuivres jouant " $\mathrm{f}$ ». Stravinsky pourtant et d'autres continueront à jouer l'ancienne disposition dans toute sa verdeur! Mais Craft ira même jusqu'à attribuer à Stravinsky l'intention, manifestée apparemment en 1943, d'éliminer complètement le mi bécarre (tout comme le doublage des parties de cors) ...5

Le tripotage de ce passage ne s'arrêtera pas là. Dès 1921 les points d'orgue sont éliminés, les accords tenus transformés en rondes et rondes pointées respectivement et la mesure originale de $2 / 2(4 / 4$ dans la réduction pour piano) modifiée en $3 / 2$. Le plus étonnant cependant n'intervient qu'en 1967, alors que la directive "Ritenuto pesante. A tempo », défigurée d'ailleurs en 1951 en un invraisemblable "a tempo ritenuto pesante " disparaît complètement, le triolet se voit affecté de staccati et les accords tenus sont à exécuter dorénavant sffp $<$ ff! Rien pourtant ne laissait dans les enregistrements de Stravinsky présager de tels changements ... Où est l'authentique Stravinsky dans tout cela? ${ }^{6}$

- Dans Cercles mystérieux des adolescentes : il semble nécessaire de corriger, comme cela fut fait enfin en 1967, l'erreur de transposition de la petite trompette en ré au tout début du no. 103 de la partition. En effet, il arriva souvent à Stravinsky dans son manuscrit autographe de confondre la transposition de la trompette en ré avec celle d'une trompette en si bémol.

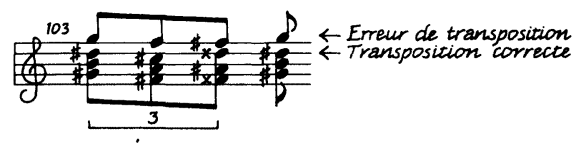

Exemple 3

Cela a donné ici la suite de notes sol/fa/fa dièse/sol, alors que l'on doit entendre, au sommet de quatre accords parfaits mineurs en position fondamentale, la ligne ré dièse/do dièse/do double dièse/ré dièse (deux tons entiers plus bas que la transcription fautive). La réduction pour piano était pourtant sans équivoque là-dessus dès le début. L'invraisemblable réside dans le fait qu'on a toujours exécuté - Stravinsky compris - la 
fausse transposition. Nous y sommes même tellement habitués que nous résistons à l'évidence logique de cette correction, car on nous prive ainsi de ce cri d'alarme strident si familier qui rompt définitivement le calme précédent en signalant le départ fracassant de la danse frénétique suivante, la Glorification de l'élue. Ce faisant, on se trouve à anticiper, en l'amenuisant considérablement, l'effet d'explosion du crescendo et des onze accords très menaçants qui suivent ... Que le compositeur luimême ne s'en soit apparemment jamais rendu compte pourrait nous en dire long sur la perspicaćité de son oreille lorsqu'il était au pupitre. A moins que la réalité ne soit beaucoup plus prosaïque et que Stravinsky, surpris puis ensuite séduit par cet accident de parcours, l'ait tout simplement endossé et laissé passer sans mot dire ... Dommage qu'il ne soit plus là pour réagir devant l'enregistrement de Simon Rattle par exemple, peut-être nous obligerait-il à " corriger " la réduction pour piano!?

Dans la Glorification de l'élue: l'examen attentif des sources fait apparaître la disparition, dès 1922 (liste d'errata), d'une alternance assez impressionnante de directives " cuivrez ", "bouché », " ouverts » pour les cors, lesquelles avaient pour effet un va-et-vient constant de coloris d'attaques des plus variés. Aussi le pizzicato des contrebasses doublant chaque « la » de la timbale dans le début et à la fin de la scène fut éliminé tacitement, tout comme fut supprimée en 1921 la directive assez exceptionnelle "frottez avec une brosse " affectant le jeu de la grosse caisse aux nos. 106 à 108 de la partition. Pourquoi Stravinsky y aurait-il renoncé? Il faut remarquer par ailleurs comment les flûtistes partent la plupart du temps en retard avec leurs appoggiatures ascendantes "en comète ", les quelles se trouvent ainsi à suivre le second ou quatrième temps de chaque mesure au lieu d'acheminer chaque " sol " carrément sur le temps. De plus, la majorité des chefs d'orchestre laissent escamoter par les cors la figure finale $\boldsymbol{\delta}$ de la cellule rythmique en $5 / 8$. Cette figure est pourtant capitale dans la connexion très étroite à établir avec la scène suivante, l'Évocation des ancêtres, laquelle s'y enchaîne, après un silence d'anticipation, avec exactement la même figure selon le même tempo :

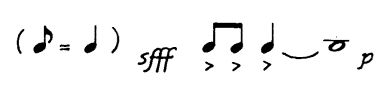


- Dans l'Évocation des ancêtres : c'est justement ce motif qui a subi en 1967 la transformation la plus étrange : on (qui?) l'a modifié en ${ }_{2}^{d} d_{3} d^{d}-\sigma$ (en le faisant doubler de timbales jouant cette fois, non pas le fa dièse répété comme auparavant, mais les hauteurs exactes du motif : fa dièse/mi/ré dièse, avec un saut ascendant de 7e)! Même si, à la vistesse $d=144$ (que très peu d'orchestre respectent), la différence pourrait passer inaperçue, étant donné qu'aucun accord ne vient sonner la ronde du ré dièse final tenu (sauf à la toute dernière répétition du motif juste avant le no. 128), il reste qu'on porte atteinte visuellement et rythmiquement au lien qui unit étroitement ces deux scènes. Il est permis de douter fortement que Stravinsky ait pu donner son assentiment à une telle défiguration. Il est bon de se rappeler également qu'à l'origine l'Évocation, du début jusqu'au no. 124 inclusivement, comportait une métrique complètement différente, beaucoup plus rapprochée et respectueuse des mesures $7 / 4$ et $8 / 4$ qui prédominaient exclusivement dans l'esquisse originale. En faisant réviser le matériel d'orchestre en 1922, Ansermet s'y montrait sensible en rétablissant les phrasés du grand rythme original moyennant des mesures longues subdivisées en conséquence par des barres de mesure en pointillés. Et pourtant, Stravinsky accepta de modifier tellement son rythme d'origine qu'on dut simplement graver à nouveau la scène entière en 1929. Cette volte-face fut-elle la sienne ou celle d'Ansermet? Il faudrait au moins pouvoir entendre l'ancienne version pour se faire une idée concrète des résultantes sonores véritables d'une telle modification.

\section{Le cas de la Danse sacrale}

La Danse sacrale illustre clairement le dilemme où Stravinsky s'est trouvé de maintenir d'un côté les articulations du grand rythme, tout en acceptant de l'autre côté, pour des raisons pratiques, de le subdiviser en unités plus petites de façon à accommoder chefs d'orchestre et instrumentistes. Il est d'ailleurs assez révélateur de voir comment, dans pratiquement tous les manuscrits autographes des œuvres, grandes et petites, de cette époque de Stravinsky, la barre de mesure est à peine visible, tracée très faiblement au plomb, furtivement, comme à regret, par pure nécessité extérieure ...

Rendre compte en détail de toutes les modifications rythmiques et instrumentales intervenues dans la Danse sacrale exigerait rien de moins qu'une monographie. Nous nous 
contenterons d'en signaler les aspects les plus marquants, en distinguant la partition de l'Édition Russe/Boosey and Hawkes - toujours rattachée à l'œuvre au complet - de celle de la révision de 1943, parue séparément chez un autre éditeur.

- La partition ERM/BH :

a) les parties centrales de cette scène (les nos. 149-166, 174-179 et 181-185) n'ont subi pratiquement aucun changement significatif, si ce n'est la redistribution à l'unisson sur quatre cors (au lieu d'un seul) du " ré " qui redouble le rythme de la percussion aux nos. 174-175;

b) les parties extrêmes par contre - le début (nos. 142-148), ses reprises (nos. 167-173, 180) et toute la coda qui s'en inspire et s'y rattache (no. 186 à la fin) - ont été modifiées

- rythmiquement, par la subdivision des mesures de $5 / 16$ et de $7 / 16$ en unités plus petites, et, ce qui est déjà plus sensible à l'audition, par le prolongement de nombreux accords - tous en double-croches à l'origine - en valeurs de croche (ce dernier détail n'est mentionné nulle part dans la documentation présentement accessible : on en constate tout simplement le fait accompli),

- instrumentalement par la disparition, tacite et sans explication aucune, des coups de cymbale et de guéro qui ponctuaient la dernière page de la partition, mais encore davantage par la suppression de très nombreux pizzicati aux cordes, surtout à partir du no. 186 et suivants, suppression qui s'est faite dans le désordre et l'incohérence les plus invraisemblables.

A ce propos précis, les observations suivantes d'Ernest Ansermet sont des plus de tout le contexte entourant ces modifications :

... Les corrections faites sur la vieille partition d'épreuve, d'après laquelle est faite l'actuelle partition gravée - ces corrections ont été faites en cours de répétitions, donc sans le soin qu'on apporte à une correction d'épreuves ... Mais voilà le point qui me préoccupe : les pizz(icati) de toute la danse sacrale. On les a supprimés en principe, parce que pressés, avec peu de cordes, et médiocres, on avait déjà bien assez à faire aux difficultés rythmiques. Je me demande sérieusement s'il faut en rester là. Après tout, il faut supposer qu'on se trouvera une fois en bonnes conditions d'exécution. Et alors, est-ce que l'écriture de ces triples cordes unis(ono) pizz(icato) et des div(isi) arco n'est pas la. 
bonne, et la/sécheresse du pizz(icato) mêlé aux hautbois ne donnera-t-elle pas un rythme plus précis que l'archet jamais assez court. L'arco perpétuel me semble (mais je ne suis qu'au pupitre) donner une sonorité uniformément grise et pâteuse, où l'intervention des pizz(icati) mettrait de la clarté et des reliefs. Ainsi aux basses ... (Ansermet in Eideldinger 1980).

En marge, presque comme de guerre lasse, Stravinsky griffonna de sa main :

Comme il n'y aura jamais dans n'importe quel orchestre que des cons, je me vois bien obligé de maintenir la supression total(e) des pizzi(cati) dans la Danse Sacrale. Il faut le noter dans l'Errata - noter la suppression des pizzi(cati) bien entendu et non cette phrase (précédente) en encre rouge (ibid.)!

Voilà qui soulève assez pertinemment le voile sur la portée capitale de cette collaboration Ansermet-Stravinsky des années vingt. Ces remarques laissent en tout cas songeur quant à la conviction réelle avec laquelle Stravinsky a accepté bien des modifications à ses ouvres, surtout - mais pas uniquement au Sacre. Quoiqu'il en soit, bien des enregistrements anciens et récents en laissent entendre de toutes les couleurs pour ce qui est du détail de la Danse sacrale. C'est même par le biais de ceux-là que l'auteur de ces lignes fut mis sur la piste d'une pareille évolution. Et il faudrait encore compléter ce tableau par les transformations assez inattendues que continue à subir même aujourd'hui la partie de timbales de cette même Danse sacrale. Ansermet en tout cas aura vu juste, même si les conséquences qu'il en a tirées dans les faits semblent aller à l'encontre de ses intuitions.

- La Danse sacrale révisée de $1943:^{8}$ on n'a pas fini de situer la portée exacte de cette étonnante révision dont on se demande d'abord si elle devait s'étendre au Sacre tout entier, mais dont l'aspect le plus insolite saute aux yeux dès la première page : c'est l'unité métrique devenue la croche au lieu de la double-croche. La danse devient ainsi une succession interminable d'accords ou de notes "monades " désarticulées. Pourtant, Stravinsky y abolit la distinction, datant de 1929, entre accords brefs et longs (est-ce un retour inconscient à l'original?), il y rétablit ici et là des pizzicati (même chose?) 
en introduisant même de nouveaux, la partie de cuivres y est orchestrée de façon plus homogène, plus uniforme et moins variée (l'orchestre ne diminue nullement de dimensions), deux accords se trouvent avoir presque complètement disparu (est-ce un oubli?), le tamtam est éliminé complètement de la section centrale (les anciens nos. 174 et ss.), timbales et grosse caisse sont articulées très différemment, les subdivisions métriques de 5 et de 7 sont souvent inversées ( 2 et 3 , au lieu de 3 et 2 , ou viceversa, etc.) et la rythmique s'y trouve parfois complètement modifiés. Il s'agit d'une véritable métamorphose qui ne semble toutefois pas aussi perceptible pour l'auditeur non averti qu'on serait porté à le croire. Beaucoup de critiques continuent à comparer les gravures nouvelles à celle que Stravinsky fit en stéréo en 1960 sans se rendre eux-mêmes compte des différences, car c'est la seule (à part les enregistrements monophoniques de Steinberg en 1953 et de Ormandy en 1955) à comporter la Danse sacrale dans son revêtement de 1943! Comment expliquer, par contre, que dans une recension comparée, faite en juin 1970, de trois enregistrements du Sacre, dont le sien de 1960 , les points que Stravinsky lui-même critique sévèrement dans sa propre gravure de la Danse sacrale, sont précisément des changements effectués en 1943 (Stravinsky-Craft 1972 : 240)? Est-ce bien lui alors qui a rédigé cette recension, parue d'ailleurs seulement après sa mort? Il est tout de même impensable qu'il ne se soit pas rendu compte en 1960 de la version de 1943 enregistrée sous sa propre direction.

Quoique sommaires, les explications qui précèdent suffisent à faire saisir comment une ouvre si bien connue comme le Sacre du Printemps est loin d'avoir livré toute sa magie sonore. Le compositeur n'est évidemment plus là pour authentifier l'exactitude de nos fouilles. De plus, les transformations et modifications dont il a été question ici - il y en aurait bien d'autres à ajouter, on s'en doute - ne portent pas essentiellement atteinte à la vitalité rythmique et sonore de base d'une ceuvre dont les effets conservent encore tout leur impact en salle de concert aujourd'hui. Mais les nombreux points soulevés laissent quand même entrevoir un potentiel sonore plus mordant, mieux articulé, plus percussif et au rythme plus serré encore que ce que l'on nous en a révélé 
jusqu'ici. Le Sacre, repris en concert et sur disque à la lumière de tous ces détails, pourrait certainement revivre un printemps renouvelé et encore plus saisissant que jamais auparavant.

\section{NOTES}

1. Ce chiffre de 64 n'inclut donc pas l'enregistrement souvenir incomplet de l'œuvre que fit Karel Ancerl en Belgique en 1971 avec l'orchestre mondial des Jeunesses Musicales, ni les deux versions "tronquées 》 de Stokowski en 1939 - la version du film " Fantasia " de Walt Disney - et de Eger en 1977, ni les quatre enregistrements parus de la réduction pour piano à quatre mains, ni l'enregistrement numérique récent d'une version américaine pour piano solo, ni enfin les deux versions pour piano mécanique (Aeolian et Pleyela) publiées au début des années 20 .

2. "As it is, however, the newly engraved Boosey and Hawkes edition dated $1967, \ldots$ besides featuring a number of emendations of very dubious authenticity ... leaves unanswered the basic question of a thorough representation of Stravinsky's wishes, as well as suggesting the question as to why the trouble should have been taken to engrave what is no more than yet another stop-gap score of this work"

3. On trouvera un examen plus détaillé de toutes ces sources dans un article de l'auteur de ces lignes : Le Sacre du Printemps, Petite histoire d'une grande partition, à paraître chez Lattès à Paris en 1982 dans une anthologie d'articles sur Stravinsky.

4. Dont une version révisée du Sacre pour orchestre réduit publiée par Robert Rudolf en 1976 chez Belwin-Mills à New York, mais dont l'exécution n'est possible qu'à l'intérieur des frontières des États-Unis.

5. Une modification qui sera suivie à la lettre par Rudolf (StravinskyCraft $1978: 531)$.

6. A titre d'information on notera qu'à l'origine la fin du Cortège du sage ne comportait que les quatre premières mesures du no. 170 de la partition. Déjà avant la création du Sacre, Stravinsky avait ajouté (à la suggestion de Monteux?) les quatre mesures suivantes qui sont pratiquement la répétition des quatre premières. Mais entre 1914-15 et 1922 les pizzicati des cordes (sauf évidemment de celles qui jouent en trilles ou en trémolo) auront été supprimés de la partition, la contrebasse s'y verra même amputée de chaque deuxième croche. Ce sont là des modifications dont il y a tout lieu de croire qu'elles répondaient à l'extrême difficulté, sinon l'impossibilité même de jouer pizzicato toutes les notes selon la vitesse prévue $d=168$. On peut toutefois se demander pourquoi Monteux n'a pas jugé de bon de suggérer d'y pourvoir dès 1913. Le jeu d'épreuves en effet s'en tint à l'original. Stravinsky s'est par ailleurs toujours plaint de n'avoir jamais réussi à faire ressortir à son goût le contrepoint des flûtes et violons (trilles et tremolo).

7. C'est précisément d'ailleurs ce que Sam Raphling s'est cru autorisé à faire dans sa version du Sacre pour piano solo publiée par Lyra Music Company, New York, en 1975 et interprétée sur disque numérique RCA par Dickran Atamlan ...

8. La partition de poche de cette révision, publiée en $1945 \mathrm{chez}$ Associated Music Publishers de New York, est à nouveau disponible aux États-Unis depuis quelques années. On aura tout avantage à s'y reporter attentivement. 


\section{RÉFÉRENCES}

EIDELDINGER, J.J.

1980 : «Une lettre inédite d'Ansermet à Stravinsky à propos du Sacre du printemps" (14 août 1922), Revue Musicale de Suisse Romande, XXXIII/5, 210.

SPIES, C.

1972 : «Editions of Stravinsky's Music » in Boretz, B. et Cone, E.T. éds., Perspectives on Schoenberg and Stravinsky. Revised edition. New York: The Norton Library, 250-67.

STRAVINSKY, I. et CRAFT, R.

1972 : Spring Fever»(article non signé), Themes and Conclusions, London : Faber and Faber, 234-41.

STRAVINSKY, V. et CRAFT, R.

1978 : Stravinsky in Pictures and Documents, New York : Simon and Schuster. 